\title{
BER Performance of 2x2 and 4x4 Transmit Diversity MIMO in Downlink LTE
}

\author{
Uyoata E. Uyoata \\ Department of Electrical and Electronic \\ Engineering, \\ Modibbo Adama Uni. of Tech., Yola. Nigeria
}

\author{
James M. Noras \\ School of Electrical Engineering and Computing \\ University of Bradford,UK
}

\begin{abstract}
Multi-antenna(MIMO) techniques are reported to improve the performance of radio communication systems in terms of their capacity and spectral efficiency. In combination with appropriate receiver technologies they can also provide savings in the required transmit power with respect to target bit error rate. Long Term Evolution(LTE), one of the candidates for fourth generation(4G) mobile communication systems has MIMO as one of its underlying technologies and ITU defined channel models for its propagating environment. This paper undertakes a comprehensive verification of the performance of transmit diversity MIMO in the downlink sector of LTE. It uses models built using MATLAB to carry out simulations. It is deduced that generally increasing transmit diversity configuration from $2 \times 2$ to $4 \times 4$ offers SNR savings in flat fading channels though with a user equipment moving at $30 \mathrm{~km} / \mathrm{hr}$, deploying $2 \times 2$ offers higher SNR saving below $7 \mathrm{~dB}$. Furthermore bandwidth variation has minimal effect on the BER performance of transmit MIMO except at SNR values above $9 \mathrm{~dB}$ while the gains of higher modulation schemes come with a transmit power penalty.
\end{abstract}

\section{General Terms}

Digital Communication, Telecommunication

\section{Keywords}

Transmit Diversity, LTE, Signal to Noise Ratio, ITU Channels, Bit Error Rate

\section{INTRODUCTION}

Mobile communication has come a long way; since the experiment with wireless radio by Guglielmo Marconi[1], there has been a growing race to make wireless communication at par with fixed wired line communication in terms of speed and capacity. Whereas wired telephony employs a guided channel, the dynamic and unpredictable nature of the propagation path between a mobile user and the network in mobile communication poses limitations to the efficiency of mobile communication systems. One such limitation is the rapid changing of the amplitude of the propagated signal - a phenomenon called fading[1]. Various techniques have been developed to overcome the effects of the wireless medium; techniques such as channel coding and spread spectrum have been suggested and tested to handle fading which basically arises from path loss, shadowing and multipath. Another such technique is the so-called MIMO(multiple input multiple output) which involves deployment of more than one antenna at the transmitter and/or receiver[2,3].

Mobile communication has developed from an analogue privilege to a ubiquitous system requiring collaborations between standardization bodies, service providers, developers and operators for crafting specifications. 3GPP(Third Generation Partnership Project) is one such collaboration which, building on the success of its second generation mobile interface technology(GSM), has been able to bring about an advanced, widely adopted mobile technology; the LTE (Long Term Evolution)[4]. The LTE Release 8 first deployed in 2009 has MIMO as one of its key physical layer technologies. This technical paper is an attempt to verify the performance of deploying MIMO in the LTE downlink physical layer using standard available LTE models based on LTE Release 10. It focuses on the physical layer which is concerned with radio frequency application and the performance metric of interest is the bit error rate(BER). It starts out with an introduction after which related scholarly works are reviewed to create a comparable measure. The system model is described followed by simulation results and conclusion.

Propagation channel models used in this paper are as per the ITU defined channel profiles $[5,6]$. The parameters which define these propagation channel profiles are given in Table 1 .

Table 1 Extended ITU Channel Models and Their Properties [5]

\begin{tabular}{|l|l|l|l|l|}
\hline $\begin{array}{l}\text { Channel } \\
\text { Model }\end{array}$ & $\begin{array}{l}\text { Mobile } \\
\text { Velocity } \\
(\mathbf{k m} / \mathbf{h r})\end{array}$ & $\begin{array}{l}\text { Maximum } \\
\text { Doppler } \\
\text { Shift(Hz) }\end{array}$ & $\begin{array}{l}\text { Number } \\
\text { of Taps }\end{array}$ & $\begin{array}{l}\text { Delay } \\
\text { Spread }\end{array}$ \\
\hline EPA & 2 & 5 & 6 & Low \\
\hline EVA & 30 & 70 & 9 & Medium \\
\hline ETU & 130 & 300 & 9 & High \\
\hline
\end{tabular}

Correlation between multipath components is pivotal when analysing MIMO systems as it has the potential of causing reduction or loss of SNR of up to $4 \mathrm{~dB}$ at a BER of $10^{-4}[7]$ in environments dominated by Rayleigh fading. Hence it is expected that a communication system will have increasing drop in error performance as the correlation increases from low to high or as the profile properties vary from EPA to ETU.

In [8] it is argued that spatial multiplexing is likely to be more favoured by modern systems like LTE than diversity reason being that transmit diversity underutilizes available bandwidth; moreover techniques such as link adaptivity and scheduling coupled with time and frequency selectivity make traditional diversity techniques unnecessary. For a $4 \times$ 4 MIMO LTE system, a significant spectral efficiency improvement of $17 \%$ over $1 \times 4$ SIMO LTE for static scenarios is achieved though slightly over $29 \%$ performance drop occurs when a mobility of $29 \mathrm{mph}$ is introduced [9]. Hence it is established that in deploying diversity techniques, spectral efficiency is not the target rather diversity gain is. In [10], a performance of $2 \times 2$ STBC and SM are evaluated using 
throughput and BER as metrics of measurement. In [11] the performance of MIMO in LTE is viewed from the capacity perspective.

\section{SIMULATION MODEL DESCRIPTION}

The model used for simulation is MATLAB based and it is a standard LTE model developed by MATLAB. The LTE Downlink PDSCH with Transmit Diversity model is also based on LTE Release 10 and allows for $2 \times 1,2 \times 2,4 \times 2$ and $4 \times 4$ configurations. The model generates variable sized data with the size dependent on a combination of parameters which include modulation scheme, channel bandwidth and MIMO configuration. Flat fading, EPA, EVA, ETU channels are supported and at the receiver space frequency block coding is coupled with soft decision demodulation and scrambling $[12,13]$ is used. Figure 1 shows a block diagram of a typical OFDM based system.

Transport bocks of sizes in consonance with Table 7.1.7.2.1-1 of [14] were generated. For the simulation, the generated size of the transport block depends on the chosen model parameter which includes channel bandwidth, antenna configuration, and the modulation scheme. This model uses a single transport block per transmission time interval (TTI).At this stage, a 24bit cyclic redundancy check(CRC) bit is appended to the generated transport block; this serves for error detection at the receiver[12] and is based on the generator polynomial:

$g_{C R C A}(D)=\left[D^{24}+D^{23}+D^{18}+D^{17}+D^{14}+D^{11}+D^{10}+\right.$ $D 7+D 6+D 5+D 4+D 3+D+1-------(1.0)$

The codewords are scrambled bit by bit by an exclusive OR operation with a 31 Gold scrambling in the scrambler which is a user-defined MATLAB function. This process serves to reduce interference between neighbouring cells and so the scrambling sequences defined for close by cells differ. An exception is in multicast transmission[15].
Complex symbols are created from scrambled transport blocks. The modulation schemes supported in LTE and in the model are QPSK, 16QAM and 64QAM. Blocks of modulated bits are separated into layers depending on the number of antenna ports and the multi antenna scheme. For transmit diversity, the maximum number layers is four with a maximum of a single code word. These blocks of are precoded based on fixed precoder matrices or the precoder codebook which depends on the information sent by the user equipment [16]. Layer mapping is done based on section 6.3.3.2 of [16].

LTE supports transmit diversity which is based upon the so called space frequency block coding (SFBC) and the bit error rate(BER) performance of SFBC in comparison to STBC depends on the Doppler spread and the delay spread of the propagating environment[17]. In scenarios where the Doppler spread supersedes the delay spread, SFBC wins and vice versa indicating that $\mathrm{SFBC}$ is preferable in time varying channels[18]. Generally for a $2 \times 2$ transmit diversity, the received signals $r_{1}$ and $r_{2}$ can be expressed as;

$$
\left[\begin{array}{l}
r_{1} \\
r_{2}
\end{array}\right]=\left[\begin{array}{cc}
x_{1} & x_{2} \\
-x_{2}^{*} & x_{1}^{*}
\end{array}\right]\left[\begin{array}{l}
h_{1} \\
h_{2}
\end{array}\right]+\left[\begin{array}{l}
n_{1} \\
n_{2}
\end{array}\right]-------
$$

hence the MIMO channel matrix $\mathrm{H}$ as,

$H=\left[\begin{array}{llll}h_{1,1} & h_{1,2} & \cdots & h_{1, n_{t}} \\ h_{2,1} & h_{2,2} & \cdots & h_{2, n_{t}} \\ h_{n_{r}, 1} & h_{n_{r}, 2} & \cdots & h_{n_{r}, n_{t}}\end{array}\right]------$

The precoded bits are mapped into the resource grid based on [19]. Depending on the bandwidth available for transmission, the resource grid are filled with the precoded symbols though the exact data symbols transmitted depends on the resource elements taken up by the reference signals, control signals, the synchronization signals and the broadcast channel [12]. From the resource grid filled with appropriate data bits, OFDM symbols are generated for the various antennas ports.

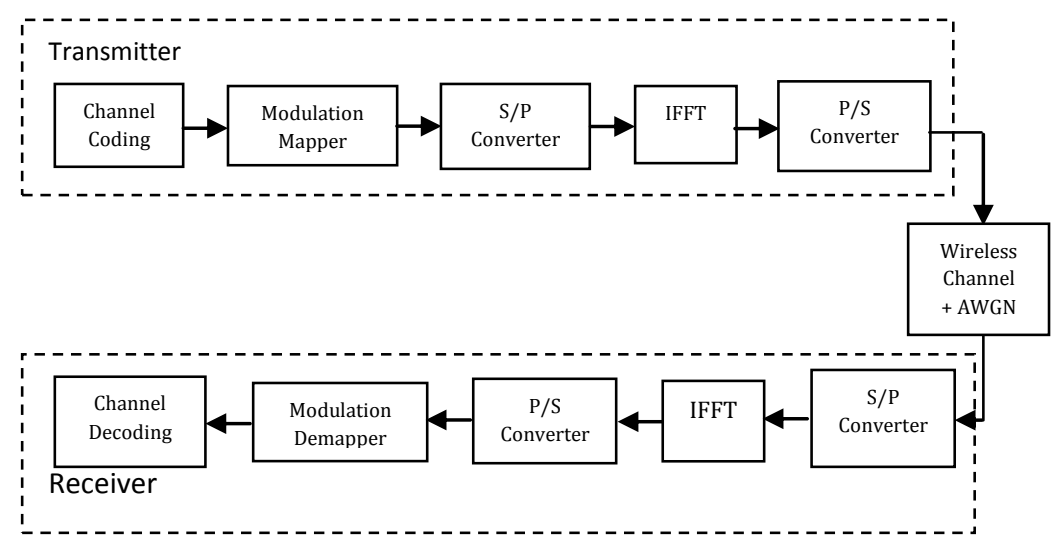

Figure 1 Block diagram of a typical OFDM based system

IFFT is performed on the filled resource grid, cyclic prefix and a DC signal are added and to the modulated data for transmission, the IFFT sizes depend on the selected bandwidth [12]. The AWGN block models a noise channel and so adds a random process to the transmitted signals. The signal to noise ratio(in decibels) selected in the model parameter dialog box is compared to the variance of the transmitted signal(converted to decibels) to provide the noise variance for the random process. For the MIMO fading channel, a user defined MATLAB function is used to implement the extended ITU channel profiles.
At the receiver the process undertaken at the transmitter is reversed for instance at the OFDM receiver, FFT is performed after which cyclic prefix and reference sequences are extracted from the received complex symbols. At the receiver, channel estimation is also carried out using Least Squares Channel Estimation and the signals equalized using an MMSE equalizer as shown in Figure 2. The LS estimator procedure is reduced to a zero forcing is described in Figure 2 [12]. In Figure 2 the pilot sequence $p$ which is locally generated at the receiver is combined with the received pilot sequence signal 
$h p$ to give the channel estimate $h$ which is combined at the equalizer to give the received signal estimate $\hat{x}$.

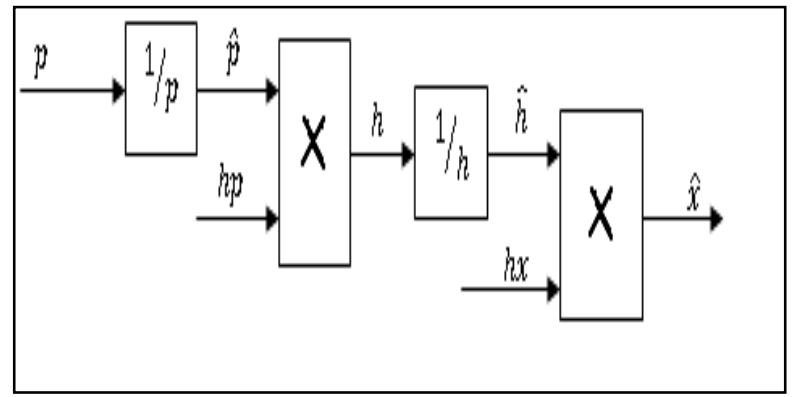

Figure 2. LS Channel Estimation Block

\section{SIMULATION RESULTS AND DISCUSSION}

The performance metrics which could be used to measure the performance of communication systems include spectral efficiency, BER, throughput, cell coverage among others. This project focused on the BER performance of transmit diversity antenna configurations of LTE Downlink models built based upon LTE Release 10 which are available via Mathworks. To obtain results, MATLAB scripts or M-files were written to load and execute the models with varied parameters of interest. The parameters varied included SNR, propagation conditions, modulation schemes and channel bandwidth. Simulations were performed for these models and the preliminary results which show the BER performance obtained by increasing the number of antennas at both transmitter and receiver. Major among the parameters used for these simulations are outlined in the Table 2.

Table 2 Simulation Parameters and values

\begin{tabular}{|l|l|}
\hline Parameter & Values \\
\hline Modulation Scheme & 64QAM \\
\hline MIMO Channel & EPA and EVA \\
\hline Maximum Doppler & $\begin{array}{l}\text { 5Hz for EPA, 70 Hz for } \\
\text { EVA }\end{array}$ \\
\hline Signal To Noise Ratio & $0-50 \mathrm{~dB}$ \\
\hline MIMO Configuration & $2 \times 24 \times 4$ \\
\hline Correlation & Low and Medium \\
\hline Channel Bandwidth & $1.4-20 \mathrm{MHz}$ \\
\hline
\end{tabular}

For the $2 \times 2$ transmit diversity MIMO configuration, the result as shown in Figure 3 indicates extremely opposing performances in the EPA profile with optimum performance obtained at moderately high speeds indicated by the $5 \mathrm{~Hz}$ maximum Doppler frequency channel condition as against a stationary user $(0 \mathrm{~Hz})$. Moreover in the vehicular channels, at SNRs below $7 \mathrm{~dB}$, performance improvement of less than $1 \mathrm{~dB}$ suffices in EVA $70 \mathrm{~Hz}$ channel whereas above $7 \mathrm{~dB}$; operating in a channel characterised by EVA $5 \mathrm{~Hz}$ conditions offers increasing reduction in required SNR for a given BER value approaching $2 \mathrm{~dB}$. Though this configuration shows a relatively poor performance for a stationary user scenario, as the required SNR exceeds $11 \mathrm{~dB}$ it becomes increasing attractive to employ this configuration in stationary scenario $(\mathrm{EPA} 0 \mathrm{~Hz}$ ) than in a channel characterised by EVA $70 \mathrm{~Hz}$ profile properties.

Furthermore in Figure 4 the performance of the $4 x 4$ system shows an upper bound delineated by the flat fading channel condition and a lower performance bound outlined by the EVA $70 \mathrm{~Hz}$ propagation profile. In between these extremes lie curves indicating the performances of the other channel profiles. The curves shows clearly that though EPA $5 \mathrm{~Hz}$ offers minimal SNR saving over EPA $0 \mathrm{~Hz}$ and EVA $5 \mathrm{~Hz}$ (both having curves which overlap up to $11 \mathrm{~dB}$ ) for SNR values below $9 \mathrm{~dB}$, above this value operating in $\mathrm{EPA} 0 \mathrm{~Hz}$ and EVA $5 \mathrm{~Hz}$ show better promise though minimal (less than 1 $\mathrm{dB})$.

Figure 5 demonstrates that the performance of lower modulated LTE systems supersedes that of higher order modulated systems[20]. Hence it can be deduced that, though employing higher order modulation schemes can offer better bandwidth efficiency[21], that result comes at the expense of poorer BER performance. A closer look at the plots reveal that though $4 \times 4$ configuration guarantees diversity gain a combination $2 \times 2$ configuration and 16QAM provides better SNR saving than $4 \times 4$ and 64QAM therefore adaptive modulation may be an option to efficiently enjoy trade-off between diversity gain and the bandwidth efficiency[22]

For the simulations to investigate if the bandwidth variations could affect the BER performance of LTE communication system, the SNR values were varied for select bandwidths $(1.4 \mathrm{MHz}, 5 \mathrm{MHz}, 15 \mathrm{MHz})$ out of the six specified bandwidths, this was done to give valuable spacing in between the plots. From the plots in Figure 6, varying the bandwidth showed insignificant SNR saving and no BER improvement except at SNR values above $17 \mathrm{~dB}$, when using a channel of $15 \mathrm{MHz}$ offers $2 \mathrm{~dB}$ and $3 \mathrm{~dB}$ savings over $1.5 \mathrm{MHz}$ and $5 \mathrm{MHz}$ bandwidths respectively for a $4 \times 4$ MIMO. Moreover, for a $2 \times 2$ MIMO there was no significant SNR saving. The result shows that at higher SNR values $(>9 \mathrm{~dB})$, there is diversity gain in deploying $4 \mathrm{x} 4$ in $15 \mathrm{MHz}$ more than in others and at SNRs above $12 \mathrm{~dB}$ using $2 \times 2$ in $15 \mathrm{~Hz}$ bandwidth offers a saving in SNR though that saving is $\operatorname{minimal}(<1 \mathrm{~dB})$. 


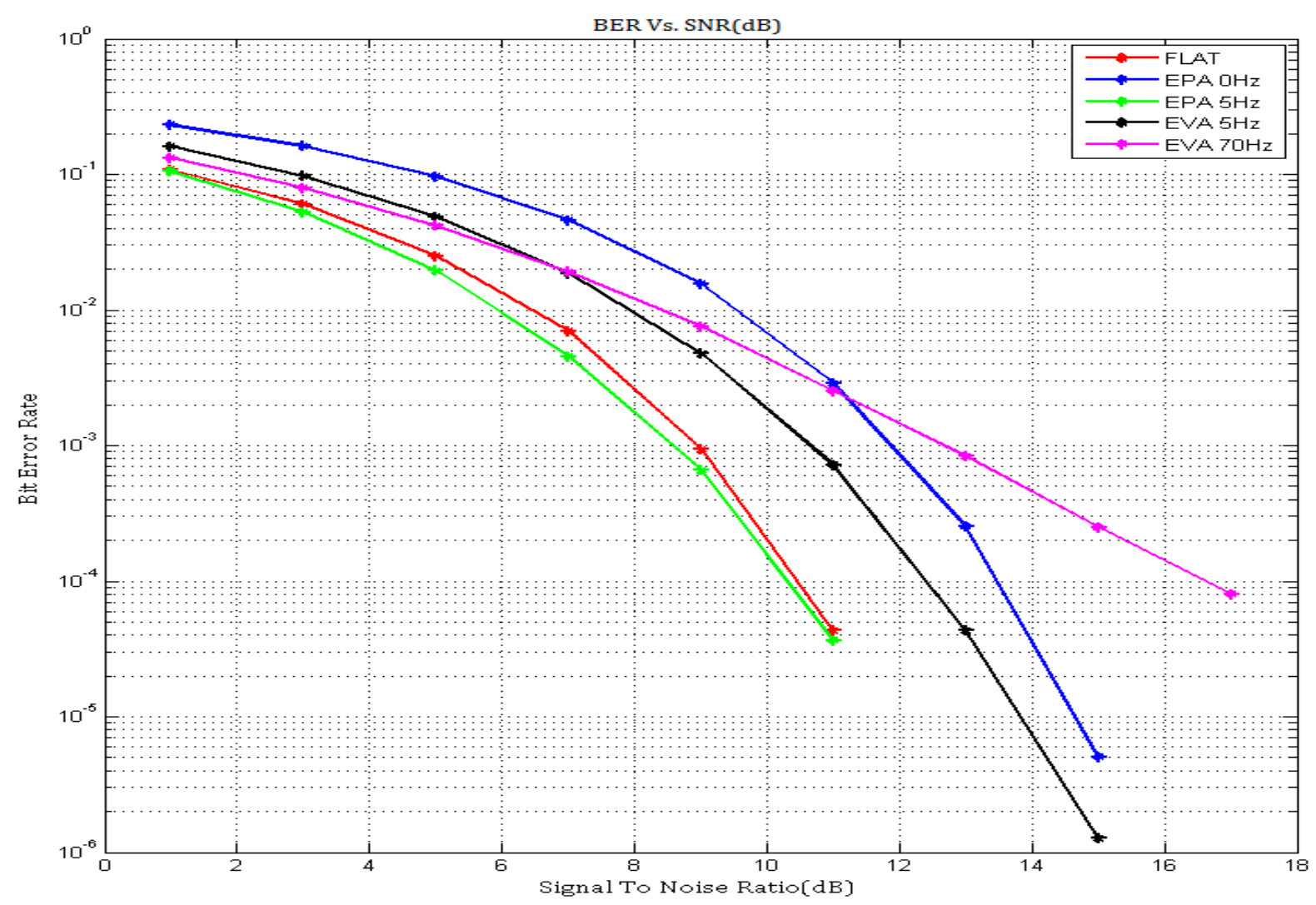

Figure 3 BER Performance of $2 \times 2$ Transmit Diversity Under ITU Channels

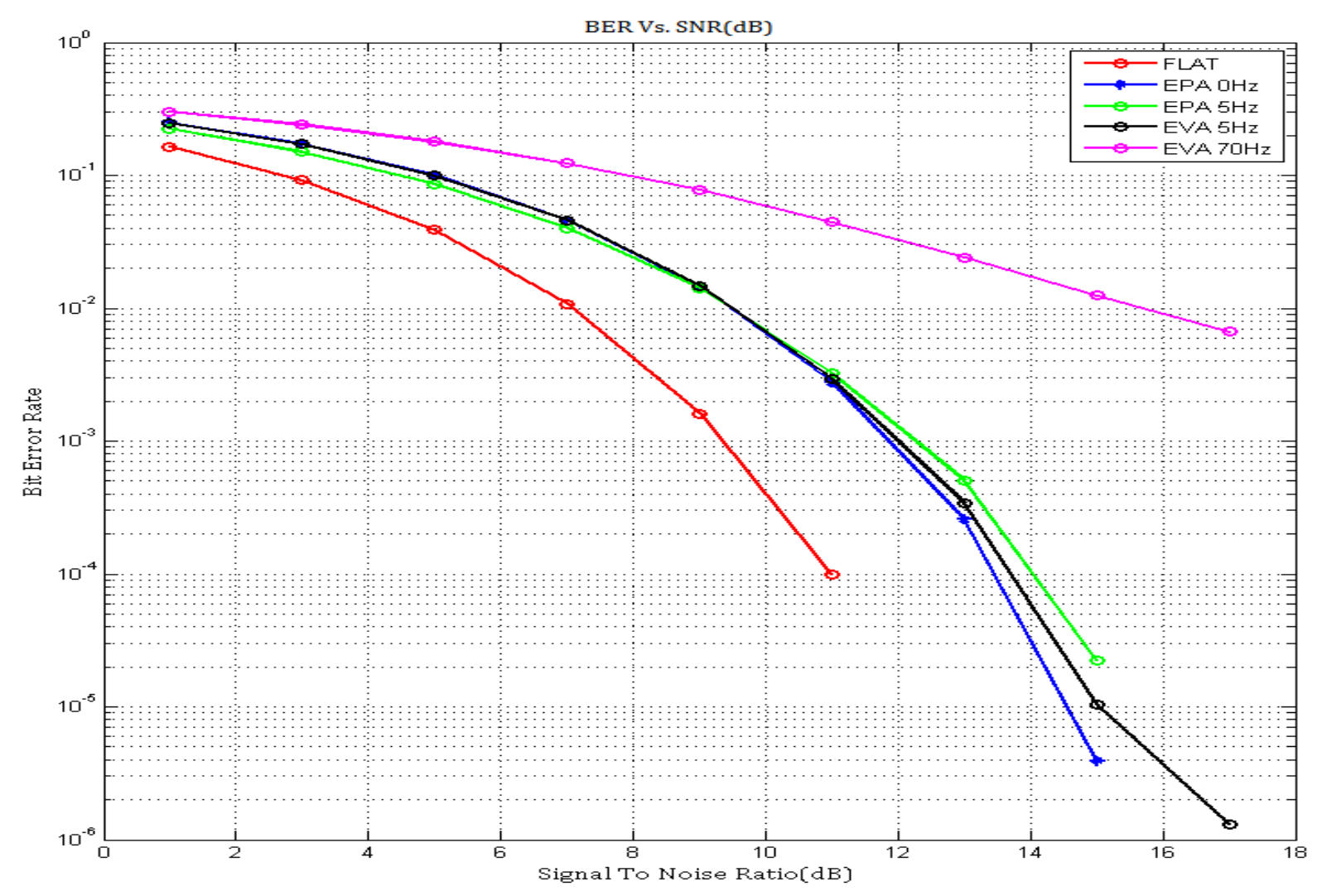

Figure 4 BER Performance of $4 \times 4$ Transmit Diversity Under ITU Channels 


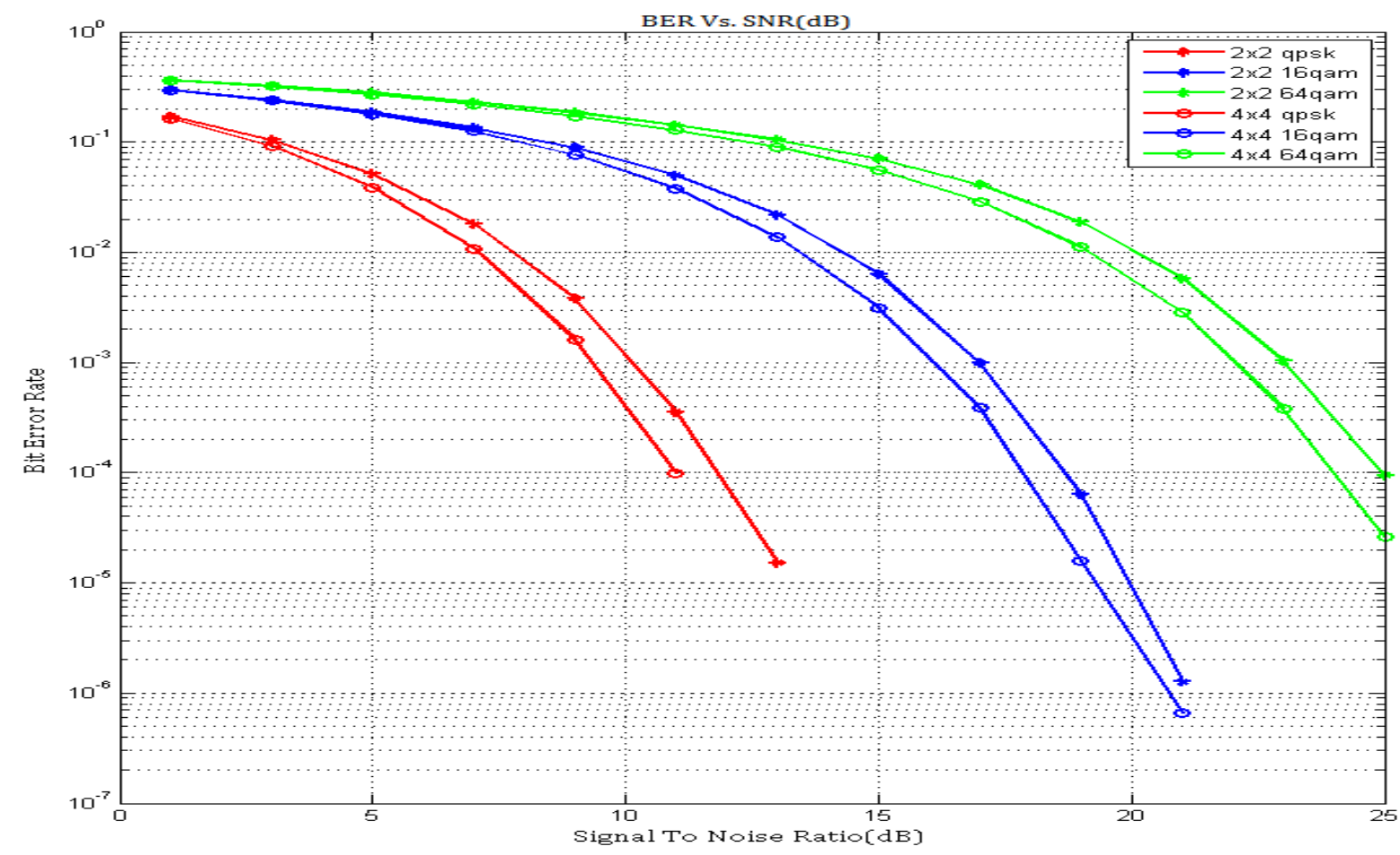

Figure 5 BER Performance of Transmit Diversity MIMO Under Various Modulation Schemes

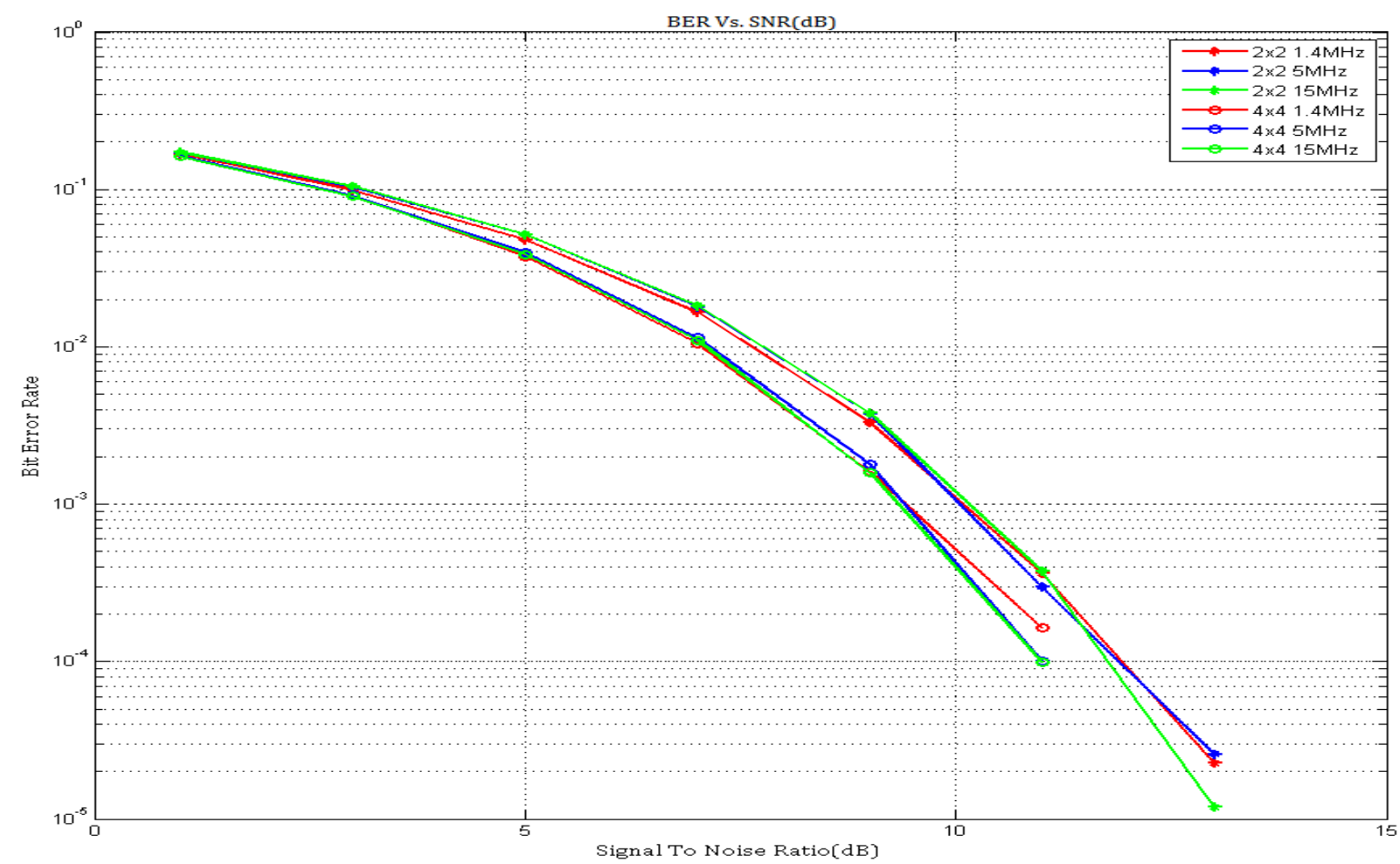

Figure 6 BER Performance of Transmit Diversity MIMO Under Various Channel Bandwidths

\section{CONCLUSION}

Generally it can be concluded that MIMO improves BER performance of LTE via diversity gain. The performance of MIMO also depends on the user equipment speed which is relates to the Doppler frequency, the target modulation scheme and less on the bandwidth chosen. Furthermore proximity to the eNodeB also determines the BER performance of a selected MIMO configuration. More specifically the conclusions obtained from this project are as follows;

1. $4 \times 4$ transmit diversity offers same target BER as $2 \times 2$ at lower SNRs

2. Deploying $2 \times 2$ transmit diversity in a $2 \mathrm{~km} / \mathrm{hr}$ scenario offers up to $2 \mathrm{~dB}$ SNR saving with respect to the flat 
fading channel. Moreover a user equipment at $30 \mathrm{~km} / \mathrm{hr}(\mathrm{EVA} 70 \mathrm{~Hz})$ is considerable in $2 \times 2$ transmit diversity MIMO below 7dB.

3. Increasing speed of user equipment up to $30 \mathrm{~km} / \mathrm{hr}$ severely deteriorates the BER performance of a $4 \times 4$ transmit diversity.

Channel estimation techniques have been proposed to affect communication system performance and so comparative analysis of MIMO using different channel estimation technique could be a viable extension of this research work.

\section{REFERENCES}

[1] Goldsmith, A Wireless Communications. New York: Cambridge University Press, 2005

[2] Foschini, J and Gans, M, 1998.On limits of wireless communications in a fading environment when using multiple antennas, Wireless Personal Communications[Online].vol.6 (3): pp. 311-335. Available: Springer Link, DOI: 10.1023/A:1008889222784. [Accessed: O4 Aug. 2013]

[3] Telatatar, I. Capacity of Multi-Antenna Guassian Channels, European Transactions on Telecommunication.[Online]. vol. 10(6), pp. 585-595, Available:, Wiley Online Library, DOI: 10.1002/ett.4460100604. [Accessed: 04 Aug. 2013]

[4] 3GPP (2013). About 3GPP. [Online]. Available: http://www.3gpp.org/About-3GPP[Accessed: 07Sept, 2013]

[5] Guidelines for Evaluation of Radio Transmission Technologies for IMT-2000,Rec. ITU-R M.1225, 1997

[6] Dahlman, E., Parkvall, S and Skold, J .4G: LTE/LTEAdvanced for Mobile Broadband: LTE/LTEAdvanced for Mobile Broadband. Kidlington, Oxford:Elsevier, 2011, pp. 1- 13

[7] Veeravalli, V and Mantravadi, A "Performance analysis for diversity reception of digital signals over correlated fading channels." Presented at IEEE 49th Vehicular Technology Conference, 1999 (Volume:2)

[8] Lozano, A and Jinda, N., 2010 Transmit diversity vs. spatial multiplexing in modern MIMO systems .IEEE Transactions on Wireless Communications, [Online].vol. 9(1), pp. 186-197.Available: IEEEXplore, DOI: 10.1109/TWC.2010.01.081381 [Accessed: 06 Aug. 2013]

[9] Farajidana, A., Chen, W., Damnjanovic, A., Yoo, T., Malladi, D. and C. Lott, "3GPP LTE Downlink System Performance."Presented at IEEE Global Telecommunications Conference, 2009.GLOBECOM.[Online].Available:IEEEXplore,DOI :10.1109/GLOCOM.2009.5425327. [Accessed: 12 Aug. 2013]

[10] Kebede, G. and Paul, O. 2010. Performance Evaluation of LTE Downlink with MIMO Techniques. Unpublished Msc Dissertation.[Online]. Available: http://www.bth.se [Accessed: 18 Feb. 2014]

[11] Barayan, Y., Kostanic,I., Rukieh, K.. 2014 "Performance with MIMO for the Downlink 3GPP LTE Cellular Systems, Universal Journal of Communications and Network [ONline]:Vol. 2(2), pp. 32-39, Available: Horizon Research Publishing (HRPUB)
[12] The MathWorks Inc., (2013). LTE PHY Downlink with Spatial Multiplexing.[Online]. Available: http://www.mathworks.co.uk. [Accessed: 2 Jul. 2013]

[13] The MathWorks Inc., (2013). LTE Downlink PDSCH with Transmit Diversity.[Online]. Available: http://www.mathworks.co.uk. [Accessed: 2 Jul.2013]

[14] ETSI LTE; Evolved Universal Terrestrial Radio Access(E-UTRA); Physical layer procedures, 3GPPTS 36.213 v10.0.0 (2010-12), Release 10, 2013

[15] Dahlman, E., Parkvall, S., and Skold, J. 4G: LTE/LTEAdvanced for Mobile Broadband: LTE/LTE-Advanced for Mobile Broadband. Kidlington, Oxford:Elsevier, 2011, pp. 1- 13

[16] ETSI LTE; Evolved Universal Terrestrial Radio Access(E-UTRA); Physical channels and modulation,3GPP TS 36.211 version 10.7.0 Release 10, 2013.

[17] Suto, K and Ohtsuki,T "Performance evaluation of space time frequency block codes over frequency selective fading channels." Presented at 2002 IEEE 56th Vehicular Technology Conference, 2002.Proceedings.VTC2002Fall. (Volume:3).[Online].A vailable: IEEEXplore, DOI: 10.1109/VETECF.2002.1040459 [Accessed: 25 Feb. 2014]

[18] Lee, K., Kim,Y, Lee, N and Kang, J “Adaptive switching between space-time and space-frequency block coded OFDM systems" Presented at IEEE Military Communications Conference, 2008.MILCOM2008. [Online].Available: IEEEXplore, DOI10.1109/MILCOM.2008.4753221[Accessed:25 Feb. 2014]

[19] ETSILTE; Evolved Universal Terrestrial Radio Access(E-UTRA);Base station radio transmission and reception (Release 10)", 3GPPTS 36.104 v10.0.0(201009)

[20] Lima,M.V.S Gussen, C.MG., Espindola, B.N., Ferreira,T.N. Martins, W.A., and Diniz, P.S.R."Opensource physical layer simulator for LTE systems."Presented at IEEE International Conference onAcoustics,Speech and Signal Processing (ICASSP), 2012.[Online]. Available: IEEEXplore, DOI:10.1109/ICASSP.2012.6288494 [Accessed: 18 Feb.2014]

[21] Wang, Z., Xie,X., Zhao, J., Tong, Z. and Yang, X "Performance analysis of different modulation schemes for coherent optical OFDM systems."Presented at International Conference on Communications and Mobile Computing (CMC), 2010 (Volume:2). [Online].Available: $\quad$ IEEEXplore, DOI: 10.1109/CMC.2010.289.[Accessed: 20 Feb.2014].

[22] Jing, S., Yan, G., Zhen, L., Yi, M. and Junde, S "Performance study of adaptive modulation/coding in MIMO-OFDM system." Presented at Canadian Conference on Electrical and Computer Engineering, 2003. IEEE CCECE 2003. (Volume:3 ). [Online]. Available: IEEEXplore, DOI: 10.1109/CCECE.2003.12262 